Ann. Zootech., I964, 13 (2), 213-2I6.

\title{
COMPARAISON DE QUATRE MÉTHODES DE MESURE DE L'ÉPAISSEUR DES \\ TISSUS ADIPEUX SOUS-CUTANÉS CHEZ LE PORC VIVANT
}

\author{
B. L. DUMONT et S. DESTANDAU ( $\left.{ }^{1}\right)$ \\ Laboratoire de Recherches sur la Viande, \\ Centre national de Recherches zootechniques, Jouy-en-Josas (Seine-et-Oise).
}

\section{SOMMAIRE}

Sur 24 porcs de $100 \mathrm{~kg}$ et à 3 niveaux corporels différents, des mesures de l'épaisseur du lard ont été effectuées en utilisant la méthode radiographique, la méthode ultrasonore, le "Live-probing " et le "Lean-meter ". Les quatre méthodes comparées se classent dans l'ordre suivant quant à leur précision : méthode radiographique, méthode ultrasonore, "Live-probing " et "Lean-meter ". Les coefficients de corrélation entre les mesures effectuées sur la carcasse et les'mesures fournies par ces méthodes étaient respectivement de $+0,994,+0,986,+0,856,+0,480$.

\section{IN'TRODUC'TION}

Quatre méthodes ont été proposées pour mesurer l'épaisseur des tissus adipeux sous-cutanés chez le porc vivant :

- méthode du "Live-probing " (HAZEL, KLINE, I952), utilisant les différences de consistance existant entre le tissu adipeux et le tissu musculaire;

- méthode utilisant les différences de conductivité existant entre le tissu gras et le muscle (appareil "Lean-Meter ») ;

- méthode radiographique (Dumont et FÉvrIER, I957);

- méthode ultrasonore (DUMONT, I957, I959).

(1) Élive de $3^{\mathrm{e}}$ année à l'W. N. S. A. T. 
Les promoteurs de ces méthodes ont indiqué, pour chacune d'elles, la précision qu'on pouvait en attendre. Mais, jusqu'à maintenant, aucune étude comparative des résultats fournis par ces quatre méthodes sur le même matériel animal n'avait été réalisée. Cette note présente les résultats que nous avons obtenus en appliquant les quatre méthodes à la mesure de l'épaisseur du lard dorsal.

\section{MATÉRIEL ET MÉTHODES DE TRAVAIL}

24 porcs de $100 \mathrm{~kg}$ de poids vif ont servi à cette étude. Sur chacun d'eux, il a été procédé à la mesure du lard dorsal en utilisant successivement la méthode ultrasonore, le Lean-Meter, la réglette métallique de HAZEL et KLINE et la radiographie.

L'épaisseur de lard fut mesurée à l'aide des quatre méthodes, aux trois niveaux suivants :

${ }_{\text {I }}^{\text {re }}$ vertèbre dorsale, dernière vertèbre dorsale, dernière vertèbre lombaire.

De légères incisions de la couenne, faites lors de la première mesure (méthode ultrasonore), servaient de points de repère pour les autres mesures et permettaient de repérer ultérieurement sur la carcasse l'endroit précis où les mesures avaient été faites in vivo.

Les mesures faites par la méthode radiographique étaient prises juste au-dessus de la colonne vertébrale (cette méthode ne permettant de faire des mesures que dans cette zone), et les mesures réalisées par les autres méthodes étaient prises toutes les trois au même endroit, à I,5 cm de la ligne médiane du dos.

Une fois les mesures prises, les porcs étaient dirigés vers l'abattoir du C. N. R. Z. où ils étaient sacrifiés dans les deux heures qui suivaient.

Après abattage et fente de la carcasse. il était procédé à la mesure de l'épaisseur de lard aux endroits où avaient été pratiquées les mesures in vivo. Pour éprouver la valeur de la méthode radiographique, l'épaisseur de lard était mesurée, au niveau où la mesure avait été faite sur l'animal vivant, sur la demi-carcasse gauche et sur la demi-carcasse droite, et la moyenne de ces deux mesures était retenue comme valeur de l'épaisseur de lard à ce niveau. Pour les autres méthodes, où la mesure de l'épaisseur de lard avait été réalisée à $\mathrm{I}, 5 \mathrm{~cm}$ de la ligne médiane du dos, il était procédé à une parfente latérale de la couche de graisse, jusqu'au niveau du tissu musculaire sousjacent. Après avoir complètement incisé cette couche de graisse, nous mesurions l'épaisseur de lard à l'endroit précis où nous avions pris la mesure in vivo.

\section{RÉSULTATS ETT DISCUSSION}

L,e tableau suivant indique les caractéristiques des mesures réalisées par ces différentes méthodes. Pour tous ces calculs, nous avons regroupé les mesures réalisées aux 3 niveaux anatomiques précédemment définis.

Cette étude confirme, en ce qui concerne la précision des méthodes radiographiques et ultrasonores, les résultats antérieurs. L'une ou l'autre de ces méthodes donne d'excellents résultats. La méthode radiographique présente sur la méthode ultrasonore l'avantage de laisser un document de travail (sur lequel il est possible d'étudier, non seulement l'importance des tissus adipeux, mais également le développement osseux de l'animal). Pour cette raison, elle doit constituer la méthode de référence pour la mesure in vivo de l'épaisseur du lard. Son prix de revient élevé et les dangers qu'elle peut présenter pour les utilisateurs limitent toutefois ses possibiblités d'emploi systématique. 
I) Comparaison des résultats fournis par la mesure directe sur la carcasse et les mesures fournies par la méthode ultrasonore, le Lean-meter et le Live-probing.

a) Valeur des moyennes:

$\begin{array}{ll}\text { moyenne des mesures "carcasse " } & 3 \mathrm{I}, 55 \mathrm{~mm} \\ \text { moyenne des mesures " ultra-sons" } & 30,90 \mathrm{~mm} \\ \text { moyenne des mesures "Lean-meter" } & 4 \mathrm{I}, 9 \mathrm{I} \mathrm{mm} \\ \text { moyenne des mesures "Live-probing " } & 30,10 \mathrm{~mm}\end{array}$

b) Comparaison des moyennes:

Ultra-son/carcasse $\quad t=0,38$ différence non significative " I,ive-probing » carcasse $t=0,9 \mathrm{I}$ différence non significative "Lean-meter $) /$ carcasse $t=5,83$ différence significative au seuil $5 \%$

c) Corrélations entre les résultats obtenus par les diverses méthodes:

$$
\begin{array}{ll}
\text { "Lean-meter } / \text { carcasse } & r=+0,480 \\
\text { "Live-probing } / \text { carcasse } & r=+0,856 \\
\text { Ultra-son/carcasse } & r=+0,986
\end{array}
$$

2) Comparaison des résultats fournis par la mesure directe sur la carcasse et la mesure fournie par la radiographie.

a) Valeur des moyennes:

$$
\begin{array}{lll}
\text { moyenne des mesures " carcasse " } & 33,3 \mathrm{~mm} \\
\text { moyenne des mesures "radiographie " } & 33,2 \mathrm{~mm}
\end{array}
$$

b) Comparaison des moyennes:

radiographie/carcasse $t=0,02$ différence non significative

c) Corrélation entre résultats fournis par la radiographie et par la mesure directe sur la carcasse:

$$
r=+0,994
$$

A côté de ces deux méthodes qui, dans l'état actuel, ne peuvent être que des méthodes de laboratoire, le "Live-probing " a fourni des résultats satisfaisants qui peuvent justifier son emploi pour des appréciations grossières de l'état d'engraissement d'un individu ou pour la mesure de l'état d'engraissement moyen de lots de porcs assez nombreux. Cette méthode a pour elle, sinon sa simplicité, du moins son prix de revient insignifiant.

Par contre, le "Lean-meter " a donné des résultats médiocres. Le point qui nous a le plus frappé a été l'inconstance de sa précision. Certaines mesures effectuées par cet appareil donnaient des résultats comparables à ceux fournis par la lecture directe sur la carcasse après abattage. Mais, le plus souvent, des écarts assez importants séparaient les deux séries de mesures. Le principe de l'appareil ne nous semble pas en cause. Ses insuffisances viennent, à notre avis, de sa réalisation. Il est vraisemblable qu'en modifiant les aiguilles, on augmenterait considérablement la précision de 1'appareil. 


\author{
SUMMARY \\ COMPARISON OF FOUR METHODS OF MEASURING THE THICKNESS \\ OF SUBCUTANEOUS FAT IN THE LIVE HOG
}

Four objective measurements of fat thickness in live pigs were compared in this study. The four techniques were radiographic, ultrasonic, Lean-meter and Live-probing (ruler probe).

They were applied at three different levels in 24 live pigs. The correlations between measurements $\left(\mathrm{N}=7_{2}\right)$ made by these four methods and the " true » fat thickness measured on the carcass immediatly after slaughtering were respectively $+0,994$ for X-ray, $+0,986$ for ultrasonic, $+0,856$ for ruler probe and $+0,480$ for Lean-meter.

\title{
RÉFÉRENCES BIBLIOGRAPHIQUES
}

HAZEL L. N., KLINE E. A., I952. Mechanical measurement of fatness and carcass value on live hogs. J. Anim. Sci., 2, 313.

DUMONT B. L., FÉVRIER R., I957. Méthode de mesure radiographique de l'épaisseur des tissus adipeux sous-cutanés chez le Porc. Ann. Zootech., 6, $29-40$.

Dumont B. L., 1957. Nouzelles méthodes pour l'estimation de la qualité des carcasses sur les porcs vivants F. A. O.-F. E.Z. Réunion sur l'Épreuve de la Descendance des Porcs. Copenhague.

Dumont B. L., I 959. Mesure de l'adiposité des Porcs par la méthode d'échos ultrasonores. C. R. Acad. Agric. France, 45, 628-631.

\author{
I.N.R.A. \\ BIBLIOTHEIU: UDEE? \\ 63033 \\ CLERMONT-FL CEDEK
}

